\title{
II Jornadas Argentinas de Educación Estadística y I Jornadas Latinoamericanas de Investigación en Educación Estadística
}

\author{
Gabriela Pilar Cabrera y \\ Liliana Mabel Tauber
}

\section{Resumen}

El año 2020 fue el año que se desató la pandemia de COVID-19, lo cual implicó que todas las actividades educativas, de investigación y de divulgación se hayan desarrollado de manera virtual. Esta situación que provocó muchas complejidades en diversos ámbitos de la vida, generó un ambiente propicio para que la comunidad latinoamericana interesada en la Educación Estadística, pudiera encontrarse a la distancia para poder intercambiar experiencias de aula, enseñanzas, aprendizajes y resultados de investigaciones centradas en la Educación Estadística. El medio para este encuentro fueron las Jornadas Argentinas de Educación Estadística y Latinoamericanas de Investigación en Educación Estadística. En este trabajo presentamos una breve descripción de las actividades realizadas y principalmente, comentamos las principales conclusiones que se obtuvieron, a partir de las cuales se han generado distintos proyectos de trabajo entre esta comunidad de aprendizaje que se presentan como cierre de este artículo.

Palabras clave: Educación Estadística, pensamiento estadístico, ciudadanía crítica, formación de profesores, Alfabetización Estadística. 


\title{
II Argentine Conference on Statistical Education and I Latin American Conference on Research in Statistical Education
}

\begin{abstract}
The year 2020 was the year that the COVID-19 pandemic broke out, which meant that all educational, research and outreach activities have been developed virtually. This situation, which caused many complexities in various areas of life, created a favorable environment for the Latin American community interested in Statistical Education, to be able to find themselves at a distance to be able to exchange classroom experiences, teachings, learning and research results focused on the Statistical Education. The means for this meeting were the Argentine Conference on Statistical Education and the Latin American Conference on Research in Statistical Education. In this work we present a brief description of the activities carried out and mainly, we comment on the main conclusions that were obtained, from which different work projects have been generated among this learning community that are presented as the closing of this article.
\end{abstract}

Keywords: Statistics Education, statistical thinking, critical citizenship, teacher education, Literacy Statistics.

Nombre del trabajo: II Jornadas Argentinas de Educación Estadística y I Jornadas Latinoamericanas de Investigación en Educación Estadística

Nombre y apellido autora 1: Gabriela Pilar Cabrera Dirección completa: Universidad Nacional de Villa María, Sede Villa del Rosario, Obispo Ferreyra 411, Villa del Rosario, Córdoba, C.P.: 5963.

Lugar de trabajo: Institutos Académicos Pedagógicos (IAP) de Ciencias Básicas y Aplicadas y de Ciencias Humanas, Universidad Nacional de Villa María.

Teléfono de contacto: +54 (03573) 454744

Dir.electrónica: gabriela.pilar.cabrera@gmail.com
Nombre y apellido autora 2: Liliana Mabel Tauber Dirección completa: Facultad de Humanidades y Ciencias, Universidad Nacional del Litoral, Ciudad Universitaria, Paraje El Pozo, Santa Fe, CP. 3000. Lugar de trabajo: Departamento de Matemática, Facultad de Humanidades y Ciencias, Universidad Nacional del Litoral.

Teléfono de contacto: +54 (0342) 4575105

Dir.electrónica: estadisticamatematicafhuc@gmail.com 


\section{Introducción}

Entre el 16 de octubre y el 14 de noviembre de 2020, se desarrollaron las II Jornadas Argentinas de Educación Estadística (JAEE) y I Jornadas Latinoamericanas de Investigación en Educación Estadística (JLIEE). Las mimas fueron organizadas por los Institutos Académicos Pedagógicos (IAP) de Ciencias Básicas y Aplicadas y de Ciencias Humanas de la Universidad Nacional de Villa María (UNVM-Argentina) y avaladas por la Facultad de Humanidades y Ciencias de la Universidad Nacional del Litoral (UNL-Argentina) y por la Red Latinoamericana de Investigación en Educación Estadística (RELIEE). La coordinación general del evento estuvo a cargo de Gabriela Pilar Cabrera (Directora del Área de Promoción de la Calidad Educativa -APCE- perteneciente al IAP de Ciencias Básicas y Aplicadas) y Liliana Noemí Villoria, integrante de APCE. Además, el Comité Académico estuvo conformado por distintos expertos en Educación Estadística de Argentina, Chile, Colombia y México.

Al igual que en su primera edición de 2019, realizada en la Universidad Nacional del Litoral (UNL-Argentina), estas Jornadas se concibieron y desarrollaron como un espacio de intercambio de propuestas didácticas, divulgación e investigación de la Educación Estadística entre investigadores, profesores y estudiantes de distintos niveles educativos. Cabe aquí señalar que, en el contexto de la pandemia mundial Covid-19 y ante la imposibilidad de realizar estas jornadas de manera presencial, esta edición se desarrolló complemente virtual y nos encontró en distintas partes del mundo a educadores e investigadores interesados en fortalecer la Educación Estadística desde edades tempranas.

\section{El panel de apertura: la Educación Estadística nos convoca}

El programa contempló 22 actividades entre paneles, charlas, conferencias y conversatorios. Particularmente, el Panel de apertura «La Educación Estadística nos convoca», estuvo integrado por: Dra. Liliana Tauber (Presidenta de RELIEE - UNL-Argentina), Mg. Gabriela Pilar Cabrera (UNVM - Argentina) y Dr. Marcel David Pochulu (UNVM-Argentina). En el panel, la Dra. Tauber abrió la discusión realizando un «breve recorrido histórico presentando algunos hitos en relación a la Estadística y a la investigación en Educación Estadística» (Canal IIJAEE-IJLIEE-UNVM2020, 2020, 1m 6s). Al finalizar su exposición compartió los propósitos de la Red Latinoamericana de Investigación en Educación Estadística -RELIEE-, en los siguientes términos: «generar redes de intercambio y colaboración de trabajos; promover espacios de diálogo, debate y reflexión e incluir y promover la participación docente (Canal IIJAEE-IJLIEE-UNVM2020, 2020, 25m $4 \mathrm{~s}$ a $29 \mathrm{~m} 1 \mathrm{~s}$ ). Justamente estas jornadas se anclaron en estos objetivos y fueron una oportunidad para ampliar el desarrollo de estos espacios con colegas de Latinoamérica y España.

Siguiendo con el panel de apertura, se puso de relieve el hilo conductor que dio sentido a la trama de contenidos que fueron desplegándose al transcurrir las 22 actividades realizadas en las Jornadas, la cual queda expresada en las preguntas enunciadas por Cabrera (Canal IIJAEE-IJLIEE-UNVM2020, 2020, $5 \mathrm{~m}$ 34s): «¿Qué aporte realiza la Educación Estadística para que las y los estudiantes tengan una mejor comprensión de la sociedad y contribuyan a su transformación?». Y en esta dirección, atendiendo al llamamiento de Pinto, Tauber, Zapata-Cardona, Albert, Ruiz y Mafokozi (2017), «¿resulta el pensamiento estadísticamente crítico el hilo conductor de la Educación Estadística en todos los niveles educativos? Si es así, ¿cuáles son las condiciones de la enseñanza de Estadística para lograr este fin?» (Canal IIJAEE-IJLIEE-UNVM2020, Cabrera, 2020, 
$13 \mathrm{~m} 1 \mathrm{~s})$. En relación a esta última cuestión, Pochulu (Canal IIJAEE-IJLIEE-UNVM2020, 2020, 28m 7s a $30 \mathrm{~m} \mathrm{11s)} \mathrm{resalta} \mathrm{que} \mathrm{«en} \mathrm{el} \mathrm{diseño} \mathrm{de} \mathrm{la} \mathrm{clase} \mathrm{hay}$ tres preguntas que son fundamentales: ¿por qué es necesario y debo enseñar un determinado contenido?, ¿qué problemas resuelve? ¿qué contextos le dan sentido y significado a lo que hace el profesor?» Es así que el panel de apertura «La Educación Estadística nos convoca» inició esta trama de contenidos que a continuación resumimos a partir del listado de las actividades con los link para acceder a cada una de ellas.

\section{Trama de contenidos desarrollados en el devenir de las Jornadas}

Las distintas actividades que se desarrollaron propiciaron el debate centrado en problemas relevantes y actuales de la Educación Estadística. Así, se discutió en torno a propuestas asociadas a distintas carreras de grado y de posgrado y, en particular, se debatió sobre la formación de profesores. En otras actividades, se expusieron los problemas específicos que actualmente se presentan en el diseño del currículo de Estadística en los distintos niveles educativos, donde se pusieron en evidencia las tensiones entre las necesidades de la Educación Estadística Crítica y las dimensiones institucionales. Siguiendo el objetivo general de las Jornadas, también se propusieron talleres en los que los asistentes pudieron vivenciar variadas propuestas de enseñanza y aprendizaje de la probabilidad y la estadística. Estas actividades no solo permitieron el intercambio y el trabajo grupal entre docentes de distintos niveles educativos sino que abrieron un espacio de reflexión y metaanálisis centrado en las propuestas que los mismos docentes pudieron vivenciar.
- Panel: ¿Cómo vive la Educación Estadística en las Ingenierías y en la formación docente? Dr. Hugo Alvarado (Vicepresidente de RELIEE. Universidad Católica de la Santísima Concepción. Chile), Mg. Rosamel Sáez (Universidad del Bío Bío - Universidad Católica de la Santísima Concepción. Chile) y Dr. Jaime García García (Universidad de los Lagos. Chile. RELIEE). Dirigido a Educación universitaria de grado. https://www.youtube.com/watch?v=rQprMICfAZY

- Conversatorio: Desafios actuales en el desarrollo de cursos de Estadística en programas de posgrado. Dr. Raúl Macchiavelli (Universidad de Puerto Rico), Dr. Fernando Casanoves (Centro Agronómica Tropical de Investigación y Enseñanza. Costa Rica) y Dr. Julio DI Rienzo (Universidad Nacional de Córdoba. Argentina). Dirigido a Educación Universitaria de grado y posgrado. https://www.youtube.com/ watch?v=_P4Y6x09_VA

- Conversatorio: Debates en torno al Currículo de Estadística en la universidad. Dr. Jesús Pinto Sosa (Universidad Autónoma de Yucatán. México), Dra. Lucía Zapata-Cardona - (Universidad de Antioquía. Colombia) y Dra. Liliana Tauber (Presidenta RELIEE. Universidad Nacional del Litoral. Argentina). Dirigido a Educación Universitaria de grado. https:// www.youtube.com/watch? $v=B T Z 6$ ErinTWs\&list=T LPQMjgwNTIwMjFnApT_vmB5_A\&index=2

- Panel: Formación de profesionales de salud: valoración del aporte de la estadística al pensamiento critico. Dr. Darío Quinodoz (Centro Médico Roentgen. Clínica La Cañada, Vila María - UNVM. Argentina), Dr. Alejandro Larriestra (Universidad Nacional Río IV. UNVM. Argentina), Dr. Jorge López-Camelo (ECLAMC CEMIC - CONICET. Argentina) y Dr. Juan Gili. (Universidad Nacional de Villa María. Argentina). Este panel estuvo dirigido a Educación Universitaria de grado y posgrado. Previo a la realización del panel se realizaron entrevistas a cada experto, las cuales pueden verse en los enlaces que incluimos en las referencias y el debate sincrónico entre todos 
los participantes está disponible en https://www. youtube.com/watch?v=LVWNh5wCFGg.

- Conferencia: Modelización para la enseñanza de fenómenos estocásticos. Mgter. Adriana Magallanes (Universidad Nacional de Río IV. Argentina. RELIEE) y Dra. Cristina Esteley (Universidad Nacional de Córdoba. Argentina). Dirigido a Educación Primaria, Secundaria y Universitaria de grado y a la Formación docente. https://www.youtube.com/ watch?v=k5IMH_ne0tw

- Conferencia: Sentido de la Estadística en la formación de profesionales de las Ciencias Económicas. Mgter. Mariana González (Universidad Nacional de Córdoba. Argentina). Dirigido a Educación Universitaria de grado y posgrado. https://www.youtube. com/watch?v=j1TNWNsWBw0

- Charla: Valoración de la interacción en los entornos virtuales de aprendizaje. Mgter. Adrián Monetta Pizarro (Universidad Nacional de Córdoba. Argentina). Dirigido a Educación Universitaria de grado y posgrado. https://www.youtube.com/ watch?v=nNpT4FEi1 HI

- Conferencia: "La relevancia de la Educación Estadística en el nivel de posgrado». Dra. Mónica Balzarini (Universidad Nacional de Córdoba - Argentina). Dirigido a Educación de Posgrado. https://www. youtube.com/watch?v=QMQgC8mpzFo

- Conferencia: «Enseñanza de la probabilidad y la estadística en el aula escolar: una herramienta para enfrentar los desafios del siglo XXI». Dra. Claudia Vásquez Ortiz (Pontificia Universidad Católica de Chile-RELIEE). Dirigido a Educación Inicial y Primaria y a Formación docente. https://www.youtube. com/watch?v=AmNSo0ArCj4

- Charla: Innovación en la producción de estadísticas locales: recursos para la enseñanza de la Estadística.
Prof. Paola Salvatierra - Prof. María Eugenia VidelaProf. Jonathan Oviedo - Prof. Valeria Ribero. Centro Estadística de Villa María (Argentina) Dirigido a todos los niveles educativos y a la Formación docente. https://www.youtube.com/watch?v=qwJMcybX_xE

- Conferencia: La actividad algebraica implicada en el trabajo con tablas estadísticas. Dra. María Magdalena Gea Serrano (Universidad de Granada. España). Dirigido a Educación Inicial, Primaria y Secundaria y Formación docente. https://www.youtube.com/ watch?v=9QWGtECGYKM

- Conferencia: Reflexiones sobre perfiles de razonamientos inferenciales informales de estudiantes universitarios. Mgter. Silvana Santellán (Universidad Nacional del Litoral. Argentina. RELIEE). Dirigido a Educación Universitaria de grado y posgrado. https://www.youtube.com/watch?v=ylYQs3i3PrM

- Conferencia: El aprendizaje estadístico pilar en procesos de machine learning y Deep learning. Lic. Jorge Sagula (Universidad Nacional de Luján. Argentina). Dirigido a Educación Universitaria de grado y posgrado. https://www.youtube.com/ watch?v=fs33tVb_hIs

- Taller 1. Taller de simulaciones en R para el aprendizaje de conceptos estadísticos. Mgter. Adriana Pérez. (Universidad Nacional de Buenos Aires. Argentina. RELIEE). Dirigido a Educación Universitaria de grado y posgrado.

- Taller 2: Una situación para problematizar la construcción de la noción de probabilidad frecuencial con estudiantes de nivel secundario. Mgter. Juan José Sosa, Prof. Sebastián Michael Filipgh y Lic. Juan Ignacio Aranda (Universidad Nacional del Nordeste. Argentina. RELIEE). Dirigido a Educación Secundaria - Formación docente. https://www.youtube.com/ watch?v=Ifi_TDqTLc0 https://www.youtube.com/watch?v=emnvWgAAOT4 
- Taller 3: Coreografías didácticas para la educación estocástica en la Educación Inicial y Primaria. Mgter. Gabriela Pilar Cabrera y Dr. Marcel David Pochulu (Universidad Nacional de Villa María. Argentina). Dirigido a Educación Inicial y Primaria y Formación docente. https://www.youtube.com/ watch?v=YGY_pUMO_sU https://www.youtube.com/watch?v=JfmhNue $\mathrm{XIOc} \& \mathrm{t}=1 \mathrm{~s}$

\section{Conversatorios entre estudiantes y entre docentes de nivel inicial a superior}

Es en este punto donde cabe resaltar que si bien en las Jornadas se desarrollaron distintas actividades, el evento que consideramos más relevante fueron los conversatorios con estudiantes y docentes que se realizaron en los dos últimos días de las Jornadas. En estos conversatorios, los principales ponentes fueron los estudiantes, quienes dieron a conocer los distintos momentos que debieron atravesar para llevar adelante sus proyectos estadísticos. También tuvieron oportunidad de mostrar sus preocupaciones y sus deseos en torno a las problemáticas que abordaron. Asimismo, la riqueza del intercambio se pudo ampliar a través de las experiencias comentadas por los docentes que trabajaron de manera colaborativa con sus estudiantes.

A través de estos conversatorios se pusieron de manifiesto los aprendizajes logrados a través de los distintos procesos de trabajo por parte de estudiantes que han sido parte de propuestas centradas en el trabajo basado en proyectos. Asimismo, se evidenciaron los procesos de retroalimentación que puede lograr el docente para realizar reajustes en sus propuestas de tal modo de propiciar aprendizajes críticos y significativos.

- Conversatorio: \#Estudiantes universitarios y graduados. Diversos ponentes de Universidad Nacional de Villa María y Universidad Nacional del Litoral
(Argentina). Dirigido a todos los niveles educativos y Formación docente. https://www.youtube.com/ watch?v=bHKoC62zfGw

- Conversatorio: \#Jardines de infantes, escuelas primarias y educación de adultos. Diversos ponentes de instituciones de educación inicial y primaria de Argentina y Brasil. En el caso de los ponentes de Brasil, han tenido traducción simultánea. Dirigido a Educación Inicial y Primaria y Formación docente. https://www.youtube.com/ watch?v=x9SDae4CA4k

- Conversatorio: \#Estudiantes nivel secundario. Diversos ponentes de instituciones de educación secundaria de Argentina. Dirigido a Educación Secundaria y Formación docente. https://www. youtube.com/watch?v=5SPw8Gi9hRk

- Conversatorio: \#Conclusiones de las jornadas. Intervención de participantes y asistentes a las Jornadas.

\section{Algunas conclusiones derivadas de las discusiones suscitadas en las Jornadas}

En esta comunidad de pensamiento y de práctica (Vásquez Bronfman, 2011; Wenger, McDermontt y Snyder, 2002) que se generó en el marco de este evento, surgieron algunas ideas potentes que promueven la investigación en Educación Estadística que llegue de manera cotidiana, natural, posible y potenciadora a las aulas de todos los niveles educativos. Compartimos a continuación algunas de estas ideas: - La alfabetización estocástica requiere iniciarse desde la educación infantil ya que esta se considera un pilar fundamental del pensamiento estadístico y para ello será necesario comenzar su desarrollo desde edades tempranas.

- Si asumimos que el hilo conductor de la Educación Estadística en todos los niveles educativos, se ancla en el desarrollo de un pensar estadístico que 
se inmiscuya en un pensar crítico, será necesario promover investigaciones situadas en el aula que revelen las condiciones de enseñanza estocástica potenciadoras de ese pensar.

- Es preciso que el profesorado de todos los niveles educativos, estudiantes e investigadores sean parte de comunidades de aprendizaje colaborativos que faciliten la producción de contenido estocástico, ya sea en relación a investigaciones, generación de bases de datos, proyectos educativos-colaborativos entre instituciones, desarrollo de repositorio de relatos de situaciones-problema y escenarios de las distintas áreas del conocimiento en los que estos conocimientos estocásticos cobran sentido.

- La RELIEE se constituye en el núcleo de esta comunidad de pensamiento y de práctica, y desde allí se gestan las acciones a seguir y cuyo siguiente nuevo mojón son las III JAEE y II JLIEE, a realizarse en la UNL en 2021.

\section{Convocatorias}

Como corolario de las actividades llevadas a cabo en 2020, nos permitimos convocarlos para formar parte de estas experiencias, poder compartir sus pareceres en relación con la Educación Estadística y ser miembros activos que ayuden a mejorar la enseñanza y el aprendizaje de Estadística con el único fin de formar ciudadanos que sean críticos con la información y que puedan tomar decisiones bien fundamentadas y basadas en la evidencia (Behar y Grima, 2014).

\section{1) II Jornada Argentina de Educación Estadística} y I Jornada Latinoamericana de Investigación en Educación Estadística, 2020. A quienes no hayan podido participar de las Jornadas, los invitamos a visitar el canal de YouTube: https://www.youtube. com/channel/UCZnCSk0py1fFqqRzasTdr6w?view_ as=subscriber, en el que están disponibles todas las actividades desarrolladas (a excepción del Taller 1 con el cual hubo un problema de edición del video).
2) III Jornada Argentina de Educación Estadística y II Jornada Latinoamericana de Investigación en Educación Estadística. 11 al 13 de noviembre 2021. Invitamos a participar en la edición 2021, que se realizará de manera virtual, en la Facultad de Humanidades y Ciencias de la UNL, entre el 11 y 13 de noviembre de 2021. En esta edición pretendemos mantener tres espacios que consideramos fundamentales:

- Un espacio destinado a la formación y actualización de los profesores a través de talleres y cursos - Otro espacio destinado al intercambio y discusión, centrado en distintas líneas de investigación sobre Educación Estadística en Latinoamérica y, - el más importante, que es el espacio destinado a los estudiantes, ya que consideramos que ellos deberían ser el centro para promover la Educación Estadística y estamos convencidos de que son quienes inspiran a los profesores.

¡Los esperamos! En breve publicaremos lineamientos para la presentación de ponencias de investigación y para la presentación de trabajos de estudiantes.

3) Red Latinoamericana de Investigación en Educación Estadística -RELIEE-. Desde 2013 se ha conformado una red de docentes e investigadores interesados en promover la Educación Estadística de los ciudadanos en Latinoamérica. Desde ese año, la Red ha auspiciado y ha generado distintas actividades en pos de difundir propuestas de enseñanza y también resultados de investigación que se realizan en nuestra región. Para una descripción más detallada de su historia, se puede consultar Ruiz, Albert, Pinto, Tauber, Alvarado y Zapata-Cardona (2021). Actualmente, la RELIEE lleva adelante distintos proyectos, particularmente para 2021, se propone convocar a todos sus miembros a participar de un modo más activo a través de distintos grupos de discusión en los que se intercambiará a través de distintos ejes problemáticos en el marco de las Jornadas referidas en el punto 2 . 
Es por ello, que los convocamos a participar como miembros activos de la Red, el único requisito es ser docente y/o investigador interesado en la Educación Estadística. Para ello, deben inscribirse completando un formulario Google en: https://docs.google.com/ forms/d/e/1FAIpQLSevR8kPEOhifbDsGSS2V_ nPmgApKEAs29RAU6I2Xat0yijueg/viewform. Es importante que realicen el registro en ese formulario para que puedan acceder a información que solo será compartida a los miembros registrados y también para poder emitir una certificación. Asimismo, pueden consultar información general de la Red en: https://reliee.weebly.com/ y también pueden participar de una activa comunidad de Facebook: https://facebook.com/groups/reliee/ o directamente buscar el grupo en Facebook con el siguiente nombre: RELIEE-Red Latinoamericana de Investigación en Educación Estadística.

\section{Referencias}

Behar, R. y Grima, P. (2014). Estadística: Aprendizaje a largo plazo. Factores que inciden y estrategias plausibles. En G. Sanabria Brenes y F. Núñez Vanegas (Eds.), Actas del IV Encuentro sobre Didáctica de la Estadística, la Probabilidad y el Análisis de Datos. Costa Rica.

Cabrera, G. [IIJAEE-IJLIEE-UNVM2020] (2020, 23 de octubre). La Educación Estadística nos convoca. Panel Inaugural. Parte 2 y 3 [archivo de video] Recuperado de https://www.youtube.com/ watch?v=xvIQLsi2Xe4

Larriestra, A. [IIJAEE-IJLIEE-UNVM2020] (2020, 2 de noviembre). Entrevista Dr. Alejandro Larriestra [archivo de video]. Recuperado de https://www.youtube.com/watch?v=MVbc6RHct0A.

López-Camelo, J. [IIJAEE-IJLIEE-UNVM2020] (2020, 2 de noviembre). Dr. Jorge López-Camelo [archivo de video]. Recuperado de https://www.youtube.com/watch?v=dPb-w4PMoNY.

Pinto, J.; Tauber, L.; Zapata-Cardona, L.; Albert, A.; Ruiz, B. y Mafokozi, J. (2017). Alfabetización Estadística en Educación Superior. Acta Latinoamericana de Matemática Educativa, 30, 227-235. México: Comité Latinoamericano de Matemática Educativa.

Pochulu, M. [IIJAEE-IJLIEE-UNVM2020] (2020, 23 de octubre). La Educación Estadística nos convoca. Panel Inaugural. Parte 3. [archivo de video] Recuperado de https://www.youtube.com/ watch?v=xvIQLsi2Xe4

Quinodoz, D. [IIJAEE-IJLIEE-UNVM2020] (2020, 2 de noviembre). Entrevista Dr. Dario Quinodoz [archivo de video] Recuperado de https://www.youtube.com/watch?v=2J0AQ7BV7QA.

Ruiz, B.; Albert, A.; Pinto, J.; Tauber, L.; Alvarado, H. y Zapata-Cardona, L. (2021). Red Latinoamericana de Investigación en Educación Estadística. Necesidades, expectativas y realidades. Revista Mexicana de Investigación Educativa, 26 (88), 69-94. Recuperado de http://www.scielo.org.mx/scielo. php?script=sci_arttext\&pid=S1405-66662021000100069\&lng=es\&tlng=es.

Tauber, L. [IIJAEE-IJLIEE-UNVM2020] (2020, 23 de octubre). Charla Liliana Tauber Panel Inaugural. [archivo de video] Recuperado de https://www.youtube.com/watch?v=ir6Nhgc4_EI

Vásquez Bronfman, S. (2011). Comunidades de práctica. Educar, 47(1), pp. 51-68. Disponible en: https://www.redalyc.org/pdf/3421/342130836004.pdf

Wenger, E.; McDermott, R. y Snyder, W. (2002). Cultivating communities of practice: A Guide to managing knowledge. Boston: Harvard Business School Press. 